\title{
SEPARAZIONE DI CARICA IN MISCELE DI MATERIALI ORGANICI: VALE LA LEGGE DI COULOMB?
}

\author{
GUIDO RAOS (*) \\ Nota presentata dal s.c. Stefano Valdo Meille \\ (Adunanza del 4 aprile 2019)
}

SUNTO. - Dopo una breve introduzione ai materiali fotovoltaici organici, viene affrontato il problema della separazione di carica in questi sistemi. Nei dispositivi migliori, le eccitazioni prodotte dall'assorbimento di un fotone possono essere convertire in portatori di carica positivi e negativi (rispettivamente buche ed elettroni) con una efficienza vicina al $100 \%$. Queste efficienze sono apparentemente in contrasto con la legge di Coulomb, che porterebbe le cariche a ricombinarsi a causa della loro reciproca attrazione elettrostatica. Un modello teorico sviluppato dal mio gruppo consente di superare questa apparente contraddizione, tendendo conto di effetti quantomeccanici quali la delocalizzazione dei portatori di carica.

$* * *$

ABSTRACT. - After a brief introduction to organic photovoltaic materials, I discuss the problem of charge separation in these systems. In the best devices, the excitations produced by absorption of a photon can be converted into positive and negative charge carriers (holes and electrons, respectively) with near-100\% efficiency. Such efficiencies are apparently at odds with Coulomb's law, which would lead to change recombination due to their mutual electrostatic attraction. A theoretical model developed within my group overcomes this apparent contradiction, by taking into account quantum mechanical effects such as the delocalization of the charge carriers.

La transizione dai combustibili fossili alle energie rinnovabili è una necessità non più prorogabile a causa dei cambiamenti climatici in atto, $\mathrm{e}$

(*) Dipartimento di Chimica, Materiali e Ingegneria Chimica "G. Natta", Politecnico di Milano, Italy. E-mail: guido.raos@polimi.it 
l'energia solare sarà sicuramente uno dei principali ingredienti nel mix energetico futuro. ${ }^{1}$ Attualmente il silicio rappresenta la tecnologia più consolidata per la conversione di energia solare in energia elettrica. Tuttavia, esso presenta alcuni svantaggi, tra cui spicca in particolare l'alto costo energetico (e quindi anche economico) della produzione del silicio elementare, il peso e la rigidità meccanica dei pannelli. Diverse classi di materiali innovativi sono oggetto di ricerca, per arrivare a sostituire in tutto o in parte il silicio. Tra queste, ci sono i semiconduttori organici. Essi hanno alcune caratteristiche interessanti, quali il basso costo, la possibilità di stamparli in film sottili su substrati flessibili, la facilità di integrazione in edifici (in finestre colorate, ad esempio).

Una cella solare organica richieda la co-presenza di almeno due materiali distinti (detti accettore e donatore), all'interno del suo strato attivo (il "cuore" della cella, tipicamente con spessore di 50-100 nm). ${ }^{2}$ Per avere le necessarie proprietà semiconduttive, entrambi i materiali devono essere $\pi$-coniugati, con una alternanza di legami carbonio-carbonio singoli e doppi. Possono essere polimerici, derivati dei fullereni, o altro. Inoltre, i loro livelli energetici più esterni (HOMO per highest occupied molecular orbital e LUMO per lowest unoccupied molecular orbital) devono essere opportunamente "sfasati" in energia, in modo da facilitare la conversione di un eccitone (lo stato elettronico generato dall'assorbimento di un fotone) in una coppia elettrone-buca. ${ }^{3}$ L'elettrone (con carica negativa, $-e$ ) va sul materiale accettore, mentre la buca (con carica positiva $+e$ ) va sul materiale donatore. L'attrazione Coulombiana tra queste cariche tenderebbe a mantenerle localizzate all'interfaccia tra i due materiali, che tipicamente vengono preparati con una morfologie nanosegregata detta a bulk beterojunction. ${ }^{4}$ Una volta localizzate all'interfaccia, entro pochi nanosecondi dalla loro formazione, le due cariche tenderebbero a ricombinarsi e l'energia del fotone andrebbe dunque perduta. Ciò nonostante, è noto che le cariche possono diffondere lontano l'una dall'altra e generare una corrente elettrica (quando raggiungono gli elettrodi sui lati opposti dello strato attivo), con un'efficienza che può arrivare al 100\%.

La facilità con cui una coppia elettrone-buca può essere convertita in due cariche libere è apparentemente in contrasto con la legge di Coulomb:

$$
U(R)=-\frac{e^{2}}{4 \pi \epsilon_{0} \epsilon_{r}} \frac{1}{R}
$$


che descrive l'energia di interazione tra due cariche $\pm e$ a distanza $R$, in un mezzo con costante dielettrica relativa $\varepsilon_{r}$ (il segno negativo nell'equazione esprime il fatto che l'interazione è attrattiva). Infatti, ponendo le cariche a $R \simeq 1 \mathrm{~nm}$ (valore rappresentativo della distanza tra una molecola accettrice e una donatrice, a contatto tra di loro), $\operatorname{con} \varepsilon_{r}=3.5$ (valore tipico per i materiali organici in gioco), otteniamo un'energia di legame $U_{b} \simeq 0.4 \mathrm{eV}$. Questa energia deve essere confrontata con l'energia termica, che a temperature ambiente vale $\mathrm{k}_{B} \mathrm{~T}=0.025 \mathrm{eV}\left(\mathrm{k}_{B}\right.$ è la costante di Boltzmann e $T^{\prime \prime} T=298 \mathrm{~K}$ la temperatura assoluta). Questa rappresenta l'energia "disponibile" per la dissociazione, in base alle leggi della termodinamica statistica. Com'è dunque possibile che la dissociazione avvenga, nonostante la grande disparità tra l'energia necessaria per la dissociazione $\left(U_{b}\right)$ e quella effettivamente disponibile a temperatura ambiente?

Sono state avanzate varie ipotesi e meccanismi, per risolvere il paradosso della separazione di carica nei materiali fotovoltaici organici: ${ }^{6,7}$

- nanostrutturazione delle miscele donatore/accettore

- disordine energetico

- campi elettrici interfacciali

- formazione di eccitoni "caldi", ad alta energia

- delocalizzazione degli elettroni e buche

- alte mobilità delle cariche

- effetti di coerenza quantistica.

Senza entrare nel dettaglio di questi meccanismi, possiamo discutere brevemente l'effetto della delocalizzazione. Questa è possibile grazie alla natura $\pi$-coniugata dei materiali, ma è anche ostacolata dalla presenza di disordine (localizzazione di Anderson). ${ }^{8}$ L'interazione Coulombiana tra due cariche diffuse (rappresentate per semplicità da due distribuzione Gaussiane $\rho(\mathbf{r})$, di ampiezza $\sigma$ e a distanza $R=\|\mathbf{R}\|)$ può essere modellata come segue:

$$
U^{\prime}(R)=-\frac{e^{2}}{4 \pi \epsilon_{0} \epsilon_{r}} \int d \mathbf{r}_{1} \int d \mathbf{r}_{2} \frac{\rho\left(\mathbf{r}_{1}\right) \rho\left(\mathbf{r}_{2}-\mathbf{R}\right)}{R}=-\frac{e^{2}}{4 \pi \epsilon_{0} \epsilon_{r}} \frac{(R / \sigma)}{R} .
$$

La Fig. 1 illustra come, quando l'ampiezza o delocalizzazione delle cariche diventa confrontabile con la loro separazione $(R \leq \sigma)$, l'interazione Coulombiana risulta molto attenuata rispetto a quella tra cariche puntuali. 


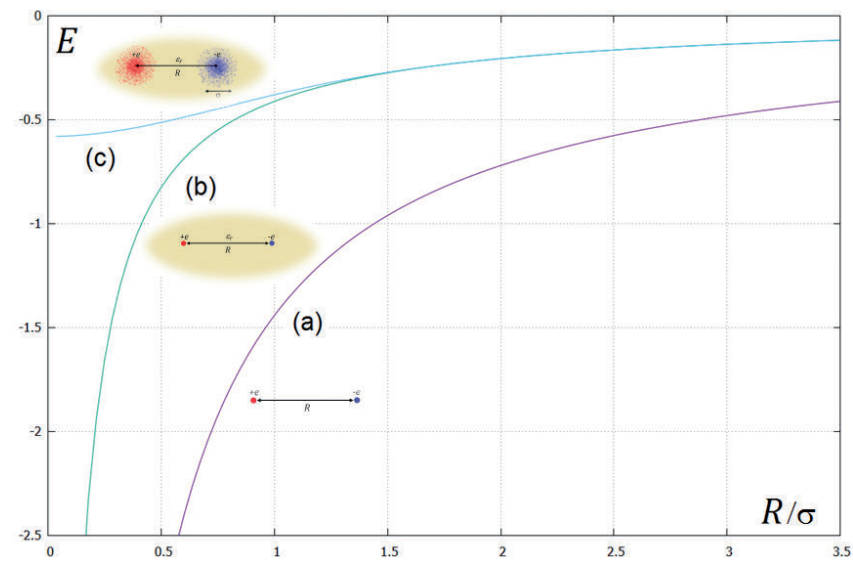

Fig. 1. Confronto dell'interazione Coulombiana tra due cariche puntuali nel vuoto (a), due cariche puntuali in un mezzo dielettrico (b), e due cariche delocalizzate in un mezzo dielettrico (c).

I moderni strumenti di calcolo consentono di simulare in modo dettagliato le interazioni all'interfaccia tra materiali donatori e accettori e, a partire da questa, calcolare le relative proprietà elettroniche.,9 Questi approcci (dinamica molecolare, chimica quantistica) richiedono ingenti risorse di calcolo e, proprio perché partono da modelli dettagliati, non sempre forniscono risposte e indicazioni generali, utili ad aumentare l'efficienza dei processi di separazione di carica. Per questo è utile studiare il problema anche con modelli semplificati, contenenti un numero limitato di parametri ed applicabili alla mesoscala $(5-30 \mathrm{~nm}$, approssimativamente). ${ }^{10}$

Negli anni passati, il mio gruppo di ricerca ha sviluppato e applicato un modello mesoscopico delle interfacce donatore-accettore. ${ }^{11,12} \mathrm{Il}$ modello riduce il problema all'essenziale, trattando ciascuna molecola accettrice o donatrice come un "sito" con due orbitali (HOMO e LUMO) e due elettroni. Le energie dei livelli energetici delle molecole sono parametrizzabili, in modo da riprodurre dati sperimentali o calcoli ab initio. L'interazione Coulombiana tra cariche, gli effetti di disordine e di delocalizzazione possono esere facilmente modulati, per verificare il loro effetto sugli stati eccitati del sistema. Il calcolo di questi stati viene fatto a livello quantomeccanico, senza approssimazioni di tipo classico. Il nostro risultato principale è che, con una scelta molto ragionevole dei parametri del modello, anche i più bassi stati eccitati di que- 
sti sistemi (eccitoni "freddi", dunque) sono descrivibili come coppie elettrone-buca delocalizzate, su lati opposti dell'interfaccia. L'energia necessaria per separare le cariche e portarle a distanza infinita è effettivamente dell'ordine dell'energia termica, $k_{B} T$. Oltre che dalla delocalizzazione, la dissociazione è favorita anche dalla formazione di un dipolo interfacciale.

Possiamo dunque rispondere alla domanda posta a titolo di questo contributo. La legge di Coulomb vale anche nei materiali organici e non è in contraddizione con l'efficienza con cui avviene la separazione di carica. Tuttavia, essa deve essere integrata con una descrizione quantomeccanica che ammette la delocalizzazione delle cariche, o la loro localizzazione nei sistemi caratterizzati da un forte disordine (nell'energia o nella sovrapposizione degli orbitali di siti diversi). Piu in generale, il nostro modello dimostra ancora una volta l'utilità di descrizioni che bilanciano la semplicità con il mantenimento di alcuni effetti fisici essenziali, nella descrizione di sistemi e fenomeni complessi.

\section{REFERENCES}

(1) Armaroli, N.; Balzani, V. Solar Electricity and Solar Fuels: Status and Perspectives in the Context of the Energy Transition. Chemistry - A European Journal 2016, 22, 32-57.

(2) Günes, S.; Neugebauer, H.; Sariciftci, N. S. Conjugated Polymer-Based Organic Solar Cells. Chemical Reviews 2007, 107, 1324-1338.

(3) Nelson, J. Polymer:fullerene bulk heterojunction solar cells. Materials Today 2011, 14, 462-470.

(4) Lu, L.; Zheng, T.; Wu, Q.; Schneider, A. M.; Zhao, D.; Yu, L. Recent Advances in Bulk Heterojunction Polymer Solar Cells. Chemical Reviews 2015, 115, 12666-12731.

(5) Park, S. H.; Roy, A.; Beaupré, S.; Cho, S.; Coates, N.; Moon, J. S.; Moses, D.; Leclerc, M.; Lee, K.; Heeger, A. J. Bulk heterojunction solar cells with internal quantum efficiency approaching 100\%. Nature Photonics 2009, 3, 297-302.

(6) Bässler, H.; Köhler, A. "Hot or cold": How do charge transfer states at the donor-acceptor interface of an organic solar cell dissociate? Physical Chemistry Chemical Physics: 2015, 17, 28451-28462.

(7) Few, S.; Frost, J. M.; Nelson, J. Models of charge pair generation in organic solar cells. Physical Chemistry Chemical Physics 2015, 17, 2311-2325.

(8) Lagendijk, A.; van Tiggelen, B.; Wiersma, D. S. Fifty years of Anderson localization. Physics Today 2009, 62, 24-29.

(9) Beljonne, D.; Cornil, J.; Muccioli, L.; Zannoni, C.; Bredas, J.-L.; Castet, F. Electronic Processes at Organic-Organic Interfaces: Insight from Modeling and 
Implications for Opto-electronic Devices. Chemistry of Materials 2011, 23, 591609.

(10) Savoie, B. M.; Jackson, N. E.; Chen, L. X.; Marks, T. J.; Ratner, M. A. Mesoscopic features of charge generation in organic semiconductors. Accounts of Chemical Research 2014, 47, 3385-3394.

(11) Raos, G.; Casalegno, M.; Idé, J. An Effective Two-Orbital Quantum Chemical Model for Organic Photovoltaic Materials. Journal of Chemical Theory and Computation 2014, 10, 364372.

(12) Casalegno, M.; Pastore, R.; Idé, J.; Po, R.; Raos, G. Origin of Charge Separation at Organic Photovoltaic Heterojunctions: A Mesoscale Quantum Mechanical View. Journal of Physical Chemistry C 2017, 121, 16693-16701. 